\title{
Estudio contrastivo del anisomorfismo cultural (Francia y España) de la terminología penal
}

\section{Contrastive study of cultural anisomorphism (France and Spain) of criminal terminology}

\author{
JORGE VALDENEBRO SÁNCHEZ \\ jorge.valdenebro-sanchez@univ-lorraine.fr \\ Université de Lorraine
}

Fecha de recepción: 16 de octubre de 2018

Fecha de aceptación: 4 de febrero de 2019

Resumen: Los ordenamientos jurídicos, a pesar de que puedan pertenecer al mismo tronco común, como es el caso de Francia y España, son propios de cada país y, por ende, distintos al resto de ordenamientos de otros países. Esto da lugar en traducción a un anisomorfismo cultural. El traductor debe ser consciente de ello para no caer en errores de traducción que imposibiliten la comprensión del lector de la lengua meta. En traducción jurídica no se traduce una realidad cultural por otra, sino que se manifiesta en la lengua meta la realidad cultural de la lengua origen. Esto se presenta como un reto para el traductor, quien deberá contar con unos amplios conocimientos culturales de ambos países y una capacidad de documentación adecuada. Por eso, el presente estudio tiene como objetivo poner de relieve la importancia de los conocimientos culturales en traducción y, más concretamente, en traducción jurídica a través del análisis de una serie de términos que provienen de la rama jurídico-penal y sus respectivas propuestas de traducción. En dicho análisis, encontraremos la explicación del concepto de la lengua origen, el contexto, la propuesta (o propuestas) traducción, la técnica de traducción que hemos empleado, así como comentarios en los que explicamos sus problemas de traducción y su anisomorfismo cultural.

Palabras clave: anisomorfismo cultural, realidades culturales, técnicas de traducción, traducción jurídica, terminología penal.

Abstract: The legal systems, even if they may belong to the same branche of law, as is the case in France and Spain, are specific in each country and, therefore, different from the rest of the legal systems of other countries. This results in translation to a cultural anisomorphism. The translator must be aware of this in order not to fall into translation errors that will make it impossible for the reader to understand the text in the target language. It is 
important to know that, in legal translation, one cultural reality is not translated by another, but that the cultural reality of the source language must be expressed in the target language. This is presented as a challenge for the translator, who must have extensive cultural knowledge of both countries and an adequate documentation capacity. Therefore, this study aims to highlight the importance of cultural knowledge in translation and, more specifically, in legal translation through the analysis of a series of terms that come from the legal-criminal branch. In this analysis, we will find the explanation of the concept of the source language, the context, the proposal (or translation proposals), the translation technique we have used, as well as comments in which we explain their translation problems and their cultural anisomorphism.

Keywords: cultural anisomorphism, cultural realities, translation techniques, legal translation, criminal terminology.

\section{INTRODUCCIÓN}

En traducción debemos saber que influyen muchos factores. Uno de ellos es el factor cultural. Esto implica que, tal y como dice Hurtado (2011), la traducción no sea únicamente una actividad en la que solo entra en juego la transposición de dos lenguas distintas, sino una comunicación intercultural. De hecho, no existen dos lenguas distintas en este mundo que estructuren la realidad de la misma manera, lo que da lugar a una disparidad lingüística, ya que un referente dado en una lengua puede no tener un equivalente exacto en otra. En consecuencia, el traductor no puede pasar por alto la importancia de los conocimientos extralingüísticos, de vital importancia en las fases de comprensión y reexpresión. Sin ellos, el traductor que solo cuente con buenos conocimientos lingüísticos podría verse en la situación de encontrarse con un texto que le resultase incomprensible, lo que daría lugar a cometer errores en el proceso traductor, como, por ejemplo, el falso sentido. Por este motivo, es fundamental el factor cultural en traducción y el trasvase de elementos culturales es uno de los mayores problemas a los que el traductor debe hacer frente. Además, podríamos decir que en traducción jurídica ( $\mathrm{y}$, más concretamente, en el ámbito penal) el factor cultural desempeña un papel clave, pues cada país organiza la realidad de un modo distinto (por ejemplo, las consecuencias legales de un acto en un país concreto no tienen por qué ser las mismas en otro país distinto). Además, debemos recordar una singularidad de la traducción jurídica: no traducimos la realidad de un país a la realidad del otro, sino que traducimos en otro idioma la realidad del país de la lengua origen. Este hecho dificulta aún más la tarea del traductor, quien debe intentar que el receptor de la lengua y cultura meta entienda la realidad de la lengua y cultura origen. En otras palabras, el traductor deberá hacer una 
propuesta de traducción apropiada en cada caso para que el lector de la lengua meta pueda comprender el concepto de la manera más clara y precisa posible.

Por tales motivos, en el presente estudio nos centramos en la importancia que tienen los conocimientos de estas diferentes realidades culturales en la traducción francés-español. Para ello, analizamos una serie de términos que provienen de la rama jurídico-penal. Hemos extraído dichos términos de la prensa francesa, de artículos de periódicos de índole penal, pues consideramos que este tipo de documentos nos proporciona una gran cantidad de terminología especializada, en un contexto determinado $\mathrm{y}$, particularmente, una gran variedad de términos que, en relación con las dos culturas estudiadas en el presente artículo (Francia y España), presentan un grado de anisomorfismo, que es por lo que este trabajo se interesa. Con la terminología extraída, hemos realizado un análisis donde podemos encontrar la explicación, el contexto, la propuesta (o propuestas) de traducción, la técnica de traducción que hemos empleado, así como comentarios en los que explicamos sus problemas de traducción y su anisomorfismo cultural.

\section{Metodología empleada en EL PRESENTE ESTUdio}

Con el fin de llevar a cabo este trabajo comparado sobre la terminología anisomórfica en el ámbito penal (Francia y España), analizamos, en primer lugar, la presa francesa para poder obtener de la terminología que pasaría a ser objeto de estudio. El motivo de haber seleccionado la prensa se debe a que es una fuente terminológica considerable que aborda ámbitos de actualidad ( $\mathrm{y}$, notablemente, temas relacionados con el ámbito penal). El contenido penal era bastante frecuente en cada uno de los artículos analizados $y$, al ser artículos franceses sobre noticias francesas, consideramos que podía ser un buen punto de partida para compararlo con la terminología española (sin entrar en el uso que hace la prensa de dicha terminología) y conseguir el objetivo de este estudio: la importancia de las diferentes realidades en traducción jurídica y el análisis del anisomorfismo cultural. En concreto, hemos recopilado 40 artículos de artículos de los años 2017-2018 de los siguientes periódicos: Le Monde, Le Figaro, La Montagne, Le Point, Le Parisien, Le Dauphine, Est Républicain, 20 Minutes, La Tribune, L'Alsace y Europe 1. El hecho de haber seleccionado estos periódicos no responde a un motivo en especial, sino al simple hecho de que se trata de prensa francesa con artículos interesantes para poder extraer la terminología que nos interesa para el presente trabajo. El proceso de selección de términos para este estudio se ha efectuado mediante el programa AntConc. No obstante, no nos hemos basado simplemente en seleccionar los términos 
o expresiones que se repetían con mayor frecuencia, sino en seleccionar aquella terminología que pertenecía al ámbito penal. Todos estos pasos dieron lugar a una primera lista terminológica. Con los términos de dicha lista, se realizó un estudio sobre cada término o expresión. El estudio consistió en el análisis conceptual de los términos franceses mediante el uso de fuentes documentales como, por ejemplo, el Código Penal (francés y español), la Ley de Enjuiciamiento Criminal y, su equivalente en Francia, el Code de procédure pénale. El objetivo de este análisis preliminar era la detección de los términos que podían presentar un problema de anisomorfismo. Después de haber realizado este estudio, se obtuvo una segunda lista más reducida con la terminología que presentaba un mayor grado de anisomorfismo cultural y que, por lo tanto, era más interesante de analizar. En el presente artículo, por motivos de espacio, hemos incluido 7 de los términos de la lista que habíamos analizado. El estudio contiene una explicación del concepto analizado, el contexto donde aparece dicho concepto, nuestra propuesta de traducción, la técnica de traducción empleada y, finalmente, un apartado de comentarios donde se comentan las diferencias, el porqué de nuestra propuesta de traducción y aspectos de interés para el traductor e intérprete de este ámbito.

\section{HACIA UNA DEFINICIÓN DE ANISOMORFISMO CULTURAL}

Cuando hablamos de anisomorfismo (en este caso, anisomorfismo cultural), debemos hacer, antes de nada, referencia al concepto de isomorfismo. El diccionario de la Real Academia de la Lengua Española lo define, en su segunda acepción, de la siguiente manera: «correspondencia biunívoca entre dos estructuras algebraicas que conserva las operaciones». Es decir, hace referencia a un concepto que pertenece al ámbito matemático y que significa, tal y como se indica en la definición, que una estructura algebraica de un conjunto tiene su correspondencia inequívoca en la segunda estructura. Por lo tanto, si traspasamos este concepto al ámbito cultural, lo que se pretende expresar es una correspondencia que se da entre dos culturas que son diferentes. Cuando hablamos de anisomorfismo estamos haciendo referencia justamente a lo opuesto de isomorfismo. Hablamos, por consiguiente, de anisomorfismo cultural en traducción (también existen otros tipos de anisomorfismo como, por ejemplo, el anisomorfismo lingüístico) cuando no existe esa correspondencia entre las dos culturas con las que se está trabajando. El anisomorfismo cultural es un concepto que todo traductor debe tener presente: su correcta resolución es uno de los mayores problemas a los que se tiene que enfrentar. Además, es algo que está muy presente en traducción jurídica, pues se trata de una traducción de ordenamientos jurídicos de diferentes países, lo que supone que la equivalencia total se va

Hikma 18 (1) (2019), $231-260$ 
a ver reducida considerablemente, ya que cada país cuenta con ordenamientos jurídicos distintos, a pesar de existir países con una base común (como es el caso de Francia y España, que se enmarcan dentro del Derecho Continental). El hecho de pertenecer a la misma familia, como es el caso de Francia y España, hace que presenten más similitudes que si comparáramos uno de estos dos países con, por ejemplo, Reino Unido, pero no significan que sean ordenamientos isomórficos.

\subsection{La traducción jurídica como una traducción de diferentes realidades}

Hurtado (2011) confirma que la traducción no se produce simplemente entre dos lenguas, sino también entre las diferentes culturas con las que estamos trabajando. Por lo tanto, la actividad traductora no se limita simplemente a traspasar lenguas aisladas de su cultura, sino que ambos elementos constituyen una sola entidad.

Tricás (2003) afirma que cada sociedad, cada cultura tiene sus propios signos de comunicación. Dichos signos nos revelan un modo de vida y pensamiento propios de ese pueblo. Sería lógico pensar que, si la lengua es el instrumento a través del cual una sociedad ve la realidad, la tarea de transmitir esa visión, desde otra perspectiva y con otros instrumentos de expresión, se anuncia un tanto complicada. De hecho, esto uno de los mayores problemas a los que el traductor debe hacer frente. Hay autores, como el caso de Catford (1965/1970: 164), que consideran incluso que es imposible traducir esos elementos culturales propios de cada país. Según este autor, se produce la intraducibilidad cultural «cuando un rasgo situacional, funcionalmente relevante para el texto en LO no existe en la cultura de la que la LT es parte» (Catford, 1965/1970: 164). No obstante, para otros autores, a pesar de las posibles variaciones que puedan existir de una lengua a otra, todas estas pueden transmitir, con mayor o menor grado, cualquier mensaje que se haya dicho en otro idioma.

Lo que sí podemos afirmar es que el proceso traslativo es una actividad compleja, donde los conocimientos culturales, además de los lingüísticos, han de estar presentes. En traducción jurídica, por lo tanto, es una de las traducciones donde la búsqueda de un equivalente plantea una dificultad extra debido al anisomorfismo entre las diferentes realidades jurídicas. Así, tal y como define Lobato (2009) entendemos la traducción jurídica del siguiente modo:

[...] por traducción jurídica entendemos que se refiere a aquel documento traducido cuya naturaleza quede encuadrada en cualquier rama del derecho, ya sean textos legislativos, convenios, 
manuales de derecho o artículos científicos sobre temas jurídicos (Lobato, 2009: 195).

Es decir, podemos entender la traducción jurídica como aquella traducción que versa sobre el derecho. He aquí uno de los mayores problemas a los que se debe hacer frente pues, como hemos mencionado anteriormente, cada país cuenta con una organización diferente en el ámbito legislativo (y, en concreto, el penal). Por consiguiente, debemos saber que las diferentes realidades con las que estamos trabajando desempeñan un papel fundamental.

\section{LA BÚSQUEDA DE UN EQUIVALENTE EN TRADUCCIÓN JURÍDICA}

Alcaraz y Hugues (2001) entienden la equivalencia como la conservación del mismo sentido en los diferentes idiomas con los que se esté trabajando. Este concepto es clave en traducción. Cada perspectiva lingüística ha intentado abordar este concepto a través de alguna propuesta enmarcada dentro de los diferentes modelos existentes como, por ejemplo, los trabajos de Nida (1975) en el estructuralismo. Para este autor, una de las condiciones indispensables para comprender el fenómeno traslativo es lo que él denomina como equivalencia semántica. Por su parte, la pragmática incluye el concepto de significado contextualizado. Este concepto incluye aspectos pragmáticos al concepto de equivalencia semántica. Desde esta perspectiva, se puede decir que lo que se busca es la equivalencia pragmática. Esta equivalencia tiene en cuenta el significado solo cuando este se encuentra dentro de un contexto. Por ese motivo, Alcaraz y Hugues (2001: 48) hablan de dos implicaciones (es decir, la implicación léxica y la implicación contextual) y dos presuposiciones (la presuposición léxica y la presuposición pragmática).

Ahora bien, la búsqueda de un equivalente en traducción jurídica no es tan evidente como parece. Para poder alcanzar tal meta, el traductor de esta especialidad debe contar una serie de parámetros que van a facilitar dicho objetivo. Tales parámetros son los siguientes (Alcaraz y Hugues, 2001: 51):

- El ordenamiento jurídico: el traductor de este ámbito debe tener conocimientos básicos de Derecho. Es conveniente destacar que no solo se debe estar familiarizado el Derecho de un país, sino con todos aquellos países cuyas lenguas son algunas con las que estamos trabajando.

- El proceso lingüístico ascendente: la comprensión del significado de las unidades del lenguaje puede ser explicado a través de dos modelos. En este subapartado se explicará el modelo ascendente.

Hikma 18 (1) (2019), $231-260$ 
Este modelo supone la identificación de las unidades mínimas de significación del lenguaje para relacionarlas gradualmente con aquellas unidades que se encuentran por encima y, de este modo, completar el texto. Es decir, se podría afirmar que la comprensión total sería la última fase de un gran proceso de descodificación de unidades lingüísticas, cada vez mayores, en las que se van incorporando. Según Alcaraz y Hugues (2001: 51), para este proceso se necesitan tres técnicas que servirá de gran ayuda al traductor. Dichas técnicas son los campos semánticos, las combinaciones léxicas y los sinónimos y antónimos.

- El proceso lingüístico descendente: el otro modelo del que hablábamos en el subapartado anterior es el descendente. Aquí, la comprensión empieza a partir de la identificación de las unidades mayores que nos resultan familiares. Por lo tanto, en este proceso, la comprensión iría de lo más general a lo más particular, reduciendo la duda hasta llegar a un acuerdo. En este modelo, el hablante se sirve de sus conocimientos y, por consiguiente, el conocimiento de los géneros jurídicos sería esencial, pues eso facilita la macroestructura generadora de expectativas que será necesaria para comprender el significado de un texto.

A priori, podría parecer fácil buscar una equivalencia entre los pares de lenguas francés-español, pues ambos idiomas pertenecen a la familia de lenguas romances y tienen un origen común. No obstante, como veremos, en el ámbito en el que nos encontramos (el jurídico) no es tan evidente dicha facilidad. Esto se debe a la diferencia en los sistemas jurídicos de ambos países, pues, aunque pertenecen la misma familia (romano-germánica), podemos encontrar un sinfín de disparidad de conceptos. El traductor debe enfrentarse constantemente a una falta de equivalente total debido al anisomorfismo de las realidades jurídicas de ambos países. Orozco (2014) afirma lo siguiente:

\begin{abstract}
La traducción jurídica lleva aparejadas múltiples dificultades, como han descrito numerosos autores, entre ellas la más destacable quizás sea el efecto jurídico que el derecho de cada país otorga a cada una de sus instituciones y por consiguiente la dificultad que entraña comunicar el contenido nocional de un término cuando no existe en la otra lengua o cultura un término que sea completamente equivalente, cosa que ocurre con mucha frecuencia, en especial si las familias jurídicas a las que pertenecen los países en cuestión son distintas (Orozco, 2014: 240).
\end{abstract}

En traducción jurídica existe un gran debate sobre qué técnicas de traducción se han de utilizar. Holl (2012: 212-213) explica que hay autores 
que prefieren el uso de equivalentes funcionales (se explicarán más adelante) o soluciones más idiomáticas en la lengua meta y otros prefieren el uso de varias técnicas para respetar ambas culturas, la original y la de llegada. Nosotros compartimos el punto de vista de Orozco (2014) y consideramos que la postura del traductor en materia jurídica es la de mediador cultural y, por consiguiente, tiene que valorar cada situación para elegir una técnica u otra.

\section{TÉCNICAS DE TRADUCCIÓN EN EL ÁMBITO JURíDICO}

En el punto anterior hablábamos acerca del equivalente en traducción jurídica y los pasos que el traductor debe dar para alcanzarlo. Uno de esos mecanismos era el uso de las técnicas de traducción. Para ello, vamos a basarnos en la clasificación propuesta por Orozco (2014), pues, después de haber analizado las diferentes taxonomías propuestas, es con la que más compartimos nuestro punto de vista. Para esta autora, la técnica de traducción facilita la labor de documentación y permite tomar decisiones más pertinentes de acuerdo con el objetivo del proceso traslativo. Esta autora divide las técnicas en función de si son equivalentes conceptuales o equivalentes lingüísticos.

\subsection{Equivalentes conceptuales}

En equivalentes conceptuales se van a englobar aquellas técnicas de traducción en las que el concepto de la lengua de origen existe también la lengua de destino.

- Equivalente total: se trata de aquellos casos en el que un concepto tiene el mismo significado y los mismos matices en los diferentes idiomas. No obstante, no es algo que suceda muy a menudo, ya que incluso equivalentes que consideramos totales pueden presentar diferencias en función del contexto en el que se use. Según Orozco (2014), la especialización del campo con el que estamos trabajando y la uniformidad o globalidad de la realidad a la que hacemos frente influye en la búsqueda de un equivalente total. Si el campo es muy especializado y uniforme, es más probable encontrar este tipo de equivalentes. En el ámbito jurídico, este tipo de equivalente lo podemos encontrar en países en los que tengan una cooficialidad de idiomas, como es el caso de Canadá, donde los documentos jurídicos se redactan en ambos idiomas.

- Equivalente natural: Šarčević (1997: 233) propone lo que denomina como «equivalentes naturales». Esto se da cuando existe ya un equivalente en la lengua de destino, que, aunque tenga matices 
diferentes, se tiene ya acuñado como equivalente. Por ejemplo, la palabra máster en español no es un equivalente total de la palabra master en francés. En Francia, el master tiene una duración de dos años, mientras que en España solo, por lo general, un año. Realmente no podemos tratarlos como equivalentes totales, pues la realidad que esconde detrás no es la misma. Sin embargo, a pesar de estos matices, lo utilizamos como equivalentes ${ }^{1}$.

- Equivalente contextual: este tipo de equivalente es el que se utiliza cuando un término de la lengua de origen tiene varias posibilidades de traducción en la lengua meta. En este caso, será el contexto el que guiará al traductor a elegir una opción u otra. Un ejemplo sería la palabra francesa acte. Dicho término, según el contexto en el que nos encontremos podrá tener una traducción u otra (acto, certificado, acta). Por ese motivo, es también muy importante conocer bien el contexto y el tipo de texto para poder encontrar ese equivalente contextual.

- Equivalente funcional: se trata por excelencia del equivalente característico en traducción jurídica. Cuando no existe un equivalente en la lengua de destino, se suele utilizar el equivalente funcional; es decir, «se busca una unidad microtextual en la cultura de llegada que cumpla la misma función que desarrolla la unidad de la lengua original en su cultura» (Orozco, 2014: 251). Por ejemplo, en el ámbito de sucesiones, podríamos decir que testamento abierto es un equivalente funcional de testament authentique. Cumple características similares, pero son diferentes. No obstante, en español, testamento abierto es lo más similar que se tiene a testament authentique $\mathrm{y}$, por ende, lo que se suele usar como equivalente funcional para que el lector pueda entender el significado de tal concepto y equivalentes lingüísticos.

\subsection{Equivalentes lingüísticos}

Cuando no existe la posibilidad de utilizar un equivalente conceptual en nuestra traducción (o queremos dejar claro que el término en cuestión es extranjero), podemos utilizar una de las alternativas lingüísticas que propone Orozco (2014) y que se trata de unas técnicas que ella denomina «equivalentes lingüísticos». Las técnicas de equivalencia lingüística que propone son las siguientes:

\footnotetext{
${ }^{1}$ Los ejemplos proporcionados en este apartado no se corresponden a los ejemplos dados por Orozco (2014), sino a una propuesta personal del autor del presente artículo.
}

Hikma 18 (1) (2019), $231-260$ 
- La traducción acuñada: es decir, utilizar por equivalente algo que ya está establecido por el diccionario de la cultura meta. Por ejemplo, «hace un frío de perros» sería la traducción acuñada de la expresión francesa il fait un froid de canard.

- Traducción léxica o calco: la traducción léxica o calco se utiliza en dos casos. Por un lado, cuando no existe un concepto equivalente en la cultura de llegada y se propone una traducción que resulte comprensible para el lector y que sea idiomática en la lengua meta (haciendo referencia a una realidad inexistente en la cultura de llegada) y, por otro lado, cuando optamos por esta técnica en lugar del uso del equivalente funcional. Por ejemplo, nosotros podemos mencionar «chalecos amarillos» como calco del francés de los gilets jaunes.

- Préstamo: incorporar un término o expresión de una lengua extranjera en nuestra lengua tal cual. Puede ser de dos tipos. Por un lado, préstamo puro; es decir, sin cambios (marketing), y, por otro lado, préstamo naturalizado; es decir, adaptar el término o expresión a las normas ortográficas de la lengua receptora (fútbol).

- Traducción perifrástica: esta técnica de traducción consiste en explicar el término (por ejemplo, con una nota a pie de páginas o mediante una aclaración entre paréntesis) en aquellos casos que no se dé un equivalente nocional en la cultura de llegada. Esto sucede en la traducción de certificado de notas entre distintos países. En España se puntúa sobre 10 y en Francia sobre 20. Esta diferencia hay que indicarla mediante una nota a pie de página.

- Neologismo: si el contexto y la situación comunicativa propicia a utilizar esta técnica, nos encontramos ante la posibilidad de crear una unidad microtextual a través del uso de los procedimientos que contamos con la lengua de llegada (como, por ejemplo, la composición).

\section{ANÁLISIS DEL CORPUS}

En el presente apartado realizamos un análisis contrastivo de la terminología penal seleccionada para el presente artículo. Como hemos indicado en el apartado 1 (cf. «Metodología empleada en el presente estudio»), nuestro estudio consta de los siguientes apartados:

- Explicación del concepto analizado

- Contexto en el que aparece (es decir, explicación breve de la noticia donde se encontraba el concepto)

- Propuesta (o propuestas) de traducción

Hikma 18 (1) (2019), $231-260$ 
- Técnicas de traducción empleada (cf. «Técnicas de traducción en el ámbito jurídico»)

- Comentarios

\subsection{ASSASSINAT}

\section{Explicación:}

El Código Penal francés define assassinat de la siguiente manera:

Le meurtre commis avec préméditation ou guet-apens constitue un assassinat.

Es decir, cuando se mata, de manera voluntaria, a otra persona, pero, además, el acto se realiza con premeditación. No obstante, consideramos conveniente definir también el concepto meurtre, ya que, muchas veces (sobre todo en la prensa y en lenguaje común) se usan como sinónimos cuando en realidad no lo son. Según el Código Penal francés, un meurtre sería lo siguiente:

Le fait de donner volontairement la mort à autrui constitue un meurtre (Code pénal, a. 221-4).

Por su parte, Cornu (1987) lo define de la siguiente manera:

Homicide volontaire ; terme générique désignant le fait de donner volontairement la mort à autrui (Cornu, 1987: 658).

Es decir, matar de manera voluntaria a otra persona.

En la definición de Cornu (1987) vemos que aparece la palabra homicide, la cual consideramos, igualmente, de dar su definición. Para este autor, el homicide sería lo siguiente:

Fait de donner la mort à un être humain, soit volontairement (C. pén., a. 221-1) ou même avec préméditation (a. 221-3), soit involontairement (a. 221-6) ou encore de façon casuelle, la mort pouvant être enfin la conséquence non voulue de violences volontaires (a. 227-7) (Cornu, 1987: 511). 
En definitiva, un homicide es el hecho de quitarle la vida a alguien, ya sea voluntaria o involuntariamente (por lo que tanto meurtre como assassinat serían un homicide al mismo tiempo).

\section{Contexto:}

En la ciudad francesa de Douai, un hombre mata, presuntamente, a su hija de ocho años porque quería "castigar» a su mujer, que había roto con él semanas antes a que se produjera tal hecho. El juicio que se está llevando a cabo es, por lo tanto, por un supuesto caso de assassinat.

\section{Propuesta de traducción:}

Asesinato

\section{Técnica de traducción empleada:}

Equivalente funcional

\section{Comentarios:}

En primer lugar, creemos conveniente explicar que, mientras que en España solo tenemos dos términos (asesinato y homicidio), en Francia existen tres (assassinat, meurtre y homicide). En lo concerniente a la traducción, hemos decidido optar por «asesinato», porque es el término que más se aproxima al término en francés. El Diccionario del español jurídico de la RAE define asesinato de la siguiente forma:

Delito consistente en dar muerte a otra persona con la concurrencia de circunstancias especialmente graves como la alevosía, por medio de precio, recompensa, con ensañamiento o su realización para facilitar la comisión de otro delito o para evitar que se descubra.

Como vemos, a priori, podemos ver que se asemejan lo suficiente como para traducir assassinat por asesinato. No obstante, es conveniente señalar que, a pesar de las similitudes que existen, se trata, una vez más, de un equivalente no total, sino funcional. En primer lugar, en la definición francesa, el asesinato solo incluye alevosía y premeditación, mientras que, en España, además de esto, se incluye otros factores como la recompensa o el ensañamiento, entre otros (el resto de situaciones se encuentra en la definición del presente apartado). Esto hace ya, de entrada, que ambos términos no sean equivalentes totales. Además, en España, si el asesinato se produce con más de una de las circunstancias mencionadas en la 
definición, la pena será impuesta en su mitad superior. Sin embargo, en el Código Penal francés no se menciona nada por el estilo.

En Francia, el asesinato se condena con una pena à réclusion criminelle à perpétuité cuando se comente contra un menor de quince años, ascendiente legítimo o natural o contra padres adoptivos, contra una persona de especial vulnerabilidad, contra una autoridad pública, contra pareja o ascendientes o descendientes en línea directa o cualquier persona que viva en el domicilio, contra un testigo, víctima o parte civil, por parte de una banda organizada, por la pareja de la víctima o contra una persona por su rechazo a contraer matrimonio.

En España, por su parte, la pena de prisión permanente no es exactamente igual que la de Francia. Asimismo, esta pena en el sistema español se puede aplicar cuando la víctima sea menor de 16 años o sea una persona especialmente vulnerable, cuando el hecho se cometa posteriormente a un delito de libertad sexual sobre la víctima, cuando el delito se cometa por parte de una organización criminal o cuando el reo de asesinato haya sido condenado por la muerte de más de dos personas. Podemos comprobar que, a pesar de numerosas similitudes, existen también muchas diferencias. En Francia, se enumeran muchas más situaciones que llevan al reo a la cadena perpetua, mientras que, en España, como vemos, las situaciones son mucho menos numerosas, por lo que, de nuevo, no podemos hablar de equivalentes totales.

En una traducción, en función del contexto, sería oportuno explicar brevemente (o dejar constancia de algún modo) de que no podemos tratar ambos conceptos por igual. Si en el documento ya se puede ver que las penas no son iguales, no habría falta poner nada. No obstante, en aquellos contextos en los que puede dar lugar a confusión, se podría indicar que las penas cambian en los diferentes países. 


\subsection{ATTEINTE SEXUELLE}

\section{Explicación:}

Según el artículo 227-25 del Código Penal francés, una atteinte sexuelle sería lo siguiente:

Le fait, par un majeur, d'exercer sans violence, contrainte, menace ni surprise une atteinte sexuelle sur la personne d'un mineur de quinze ans [...] (Code pénal, a. 227-25).

Es decir, el hecho de que una persona mayor de edad no utilice la violencia, presión, amenaza o sorpresa para mantener un abuso sexual con una persona menor de quince años. Es importante diferenciar una atteinte sexuelle de un viol o una agression sexuelle, pues las penas varían en función de si se trata de un asunto o de otro.

Así, un viol sería, según el artículo 223-23 del Código Penal francés, lo siguiente:

Tout acte de pénétration sexuelle, de quelque nature qu'il soit, commis sur la personne d'autrui par violence, contrainte, menace ou surprise est un viol (Code pénal, a. 223-23).

Por su parte, la página web del gobierno francés, service public, define la agression sexuelle de la siguiente manera:

L'agression sexuelle est toute atteinte sexuelle sans pénétration commise sur une victime avec violence, contrainte ou menace. Par exemple, des attouchements.

Es decir, tanto viol como agression sexuelle son, a modo general, actos en los que se atenta contra la libertad o indemnidad de la persona de manera violenta, amenaza u oposición por parte de la víctima. La diferencia reside en que el viol debe haber penetración, pues si no hay penetración, sería agression sexuelle.

\section{Contexto:}

¿Puede una persona menor de edad (11 años) mantener relaciones sexuales con una persona mayor de edad (28 años) de manera voluntaria o, por el contrario, se trata de una violación o abuso por parte 
del o de la mayor de edad? En el departamento de Valle del Oise, en 2018, un hombre de 28 ha sido juzgado por mantener relaciones sexuales con una niña de 11 años. La menor había denunciado por violación, pero los investigadores y el parquet consideraron que la niña no se había opuesto a ninguna resistencia, por lo que no se trataba de una violación, sino de, como se denomina en Francia, atteinte sexuelle (explicaremos el contenido más adelante). Decisión que causó un gran revuelo mediático, que hizo que se abriera una nueva investigación más profunda para aclarar los hechos $\mathrm{y}$, además, que se debatiese sobre reformar la edad de consentimiento sexual.

\section{Propuesta de traducción:}

Abuso sexual

\section{Técnica de traducción empleada:}

Equivalente funcional

\section{Comentarios:}

En primer lugar, tenemos que ver una diferencia con respecto a ambos países en lo relacionado a la edad de consentimiento sexual. En Francia, como se menciona en el artículo 227-25 del Código Penal francés (citado en la parte explicación), la edad de consentimiento sexual se establece a partir de los 15 años. Por su parte, en España, el artículo 183 del Código Penal establece lo siguiente:

El que realizare actos de carácter sexual con un menor de dieciséis años, será castigado como responsable de abuso sexual a un menor con la pena de prisión de dos a seis años (Código Penal, a. 183).

Por consiguiente, aquí tenemos la primera diferencia existente en ambos países. En Francia, si un mayor de edad tiene relaciones sexuales con alguien de 16 años, la pena cambia, pues supondría un castigo de tres años de prisión y 45000 euros de multa. Asimismo, debemos hablar de las penas que atañe dicho acto en los diferentes países. En Francia, tal acto está castigado con cinco años de prisión y 75000 euros de multa. No obstante, en España, la persona que realice tal acto puede ser castigado con una pena de prisión de dos a seis años. Vemos que los años de prisión no son los mismos en España y en Francia y, además, en Francia tal acto 
acarrea también una multa, mientras que en España no se menciona nada sobre multas, sino simplemente los años de prisión.

Por estos motivos explicados anteriormente, hemos de tener en cuenta que, aunque hemos traducido atteinte sexuelle por abuso sexual, la realidad nos dice que no son equivalentes totales. Hemos optado por un equivalente funcional, ya que en la cultura de llegada es el concepto que más se le parece y que, a nuestro modo de ver, el que más facilita la comprensión al lector de la lengua meta. Sin embargo, es importante que tengamos en cuenta todas las diferencias que tienen. En nuestra opinión, en un texto divulgativo como, por ejemplo, la prensa, no sería necesario dar ningún tipo de explicación al respecto, pues el objetivo es la mera comprensión del texto (sin entrar en profundidades). No obstante, si es un texto jurídico enmarcado dentro de un proceso judicial, sí que consideraríamos oportuno hacer una breve explicación (utilizando la técnica de traducción perifrástica con una nota a pie de página, por ejemplo) para evitar cualquier tipo de confusiones con ambos sistemas y dejar claro que, aunque sean conceptos "parecidos», no son conceptos sinónimos o equivalentes totales.

\subsection{COUR D'APPEL}

\section{Explicación:}

Francia metropolitana se organiza en regiones judiciales. Cada región cuenta con una Cour d'appel, que constituyen una de las instancias penales del sistema jurídico francés. Examina y verifica todo para comprobar que no hay errores. Revisa las decisiones que ya han sido juzgadas en el ámbito civil, comercial, social o penal. Puede o bien confirmar la decisión tomada por los primeros jueces o anularla de manera total o parcial. Reexamina las decisiones que provienen de los siguientes tribunaux:

- Tribunal d'instance (asuntos cuya cantidad demandada por la justicia sea superior a 4.000 euros o esté determinada)

- Tribunal de grande instance

- Tribunal de commerce

- Conseil de prud'hommes (sumas superiores a 4.000 euros)

- $\quad$ Tribunal paritaire des baux ruraux 
- Tribunal des affaires de sécurité sociale

- Tribunal de police

- Tribunal correctionnel

- De los jueces especializados como el juge d'instruction, juge de l'application des peines, etc.

La Cour d'appel se compone de magistrats ${ }^{2}$ profesionales: un primer presidente, presidentes de cámara y consejeros.

\section{Contexto:}

Michel Neyret, fue el antiguo director adjunto de la dirección interregional de la policía judicial de Lyon. En 2011 fue detenido por un supuesto caso de narcotráfico. Recurrió la pena que se le había puesto en primera instancia y ahora está a la espera de que la Cour d'appel tome decisión el día 12 de junio de 2018.

\section{Propuesta de traducción:}

Tribunal de Apelación

\section{Técnica de traducción empleada:}

Calco

\section{Comentarios:}

En el sistema penal español, el organismo más similar con el que contamos son las Audiencias Provinciales. La página web del Poder Judicial España define las Audiencias Provinciales de la siguiente manera:

Son tribunales de justicia que abarcan una provincia y tienen su sede en la capital respectiva. Son órganos colegiados con competencia en los órdenes jurisdiccionales civil y penal.

Podemos ver, en la primera parte de la definición, la primera diferencia con respecto a la Cour d'appel: provincia. España organiza su territorio en comunidades autónoma, fruto de la descentralización del gobierno. Estas comunidades autónomas gozan de, como su nombre indica, cierta autonomía con respecto al gobierno central. Cada comunidad autónoma se compone de provincias. Por su parte, Francia organiza su territorio en regiones que, aunque también fruto de la descentralización del

${ }^{2}$ Hemos decidido no traducir (en este apartado) este término porque se trata de una de las unidades que se analizan en el presente estudio. 
gobierno, tienen menos autonomía que las comunidades autónomas españolas. Además, las Cours d'appel no están presentes ni en cada departamento ni en cada región de Francia, sino que las podemos encontrar en cada una de las regiones jurídicas presentes en Francia (y que no corresponden forzosamente a las regiones territoriales de las que hemos hablado antes). Por lo tanto, no podemos decir que ambos conceptos, el francés y el español, sean equivalentes totales, pues, como acabamos de ver, detrás de ellos se esconde una diferencia cultural considerable. Por ese motivo, consideramos inoportuno traducir Audiencia Provincial por Cour d'appel, pues, como hemos mencionado, en Francia no existen las provincias y, en caso de utilizar (incorrectamente) como sinónimo provincia y departamento, vemos que tampoco existe una Cour d'appel por departamento.

\subsection{JUGE DES LIBERTÉS ET DE LA DÉTENTION}

\section{Explicación:}

Según la página web del Ministerio de Justicia de Francia, el juge des libertés et de la détention es uno de los jueces que interviene cuando los derechos de las personas peligran. Es quien se pronuncia sobre la prisión provisional (o su prolongación) y sobre la puesta en libertad.

Según Cornu (1987), el juge des libertés et de la détention sería lo siguiente:

Juridiction à juge unique instituée comme un rouage démultiplié de l'instruction dans le procès pénal pour statuer, en attribution exclusive, sur la détention provisoire (l'ordonner ou la prolonger) et sur les demandes de mise en liberté, fonction exercée au sein du tribunal de grande instance par un magistrat du siège désigné par le président de ce tribunal [...] (Cornu, 1987: 584).

En definitiva, es quien decide sobre si se ha de proceder a las medidas cautelares (sobre todo la prisión provisional de la persona que está siendo investigada) que han sido solicitadas por el instructor.

Contexto:

En agosto de desaparecía en Pont de Beauvoisin (Francia) una niña de 8 años que se llamaba Maëlys. Esta se encontraba con sus padres 
la noche que desapareció en la celebración de una boda. Estaba jugando con los demás niños que se encontraban en la celebración cuando, al momento en el que los padres decidieron marcharse, no había rostro de la pequeña. Hubo meses de investigación en el que la policía sospechaba de uno de los invitados. Más tarde, a pesar de no haber encontrado aún el cuerpo, Nordahl Lelandais es detenido como autor del delito (por la gran cantidad de indicios que había). Él negaba todo el tiempo el delito hasta que la policía encontró restos de sangre en el maletero del coche de Lelandais que coincidían con la de la pequeña. Por este motivo, decidió contar que la había matado de forma involuntaria y condujo a la policía al lugar donde había ocultado el cadáver. Ahora la Cour d'appel de Grenoble ha rechazado la puesta en libertad del presunto asesino. Una propuesta que ya había sido rechazada por el juge des libertés et de la détention.

\section{Propuesta de traducción:}

Juez en materia de libertades y prisión preventiva

\section{Técnica de traducción empleada:}

Traducción perifrástica

\section{Comentarios:}

En primer lugar, aquí tenemos un problema de gran envergadura. El juge des libertés et de la détention es una figura francesa que no existe en España. Estamos aquí ante un claro ejemplo de anisomorfismo cultural. En España, la figura que decide sobre la prisión preventiva, su prolongación o su puesta libertad es el juez. No existe una figura específica para ello. No obstante, no consideramos oportuno traducirlo simplemente por la palabra «juez», ya que es un concepto más genérico que el término francés. Además, es importante saber que, como hemos dicho anteriormente, en España, según si el órgano en cuestión es unipersonal o colegiado, hablamos de juez o magistrado. Una diferencia que, en Francia, no se daba. El CNRTL define juge de la siguiente manera:

Magistrat chargé par l'autorité publique de rendre la justice dans les tribunaux de première ou grande instance (p. oppos. aux Conseillers des Cours).

Vemos que la misma definición utiliza la palabra magistrat para definir al juge. $Y$ es que en Francia un juez es un magistrado al mismo tiempo, mientras que en España el magistrado es una categoría superior 
al juez, que se adquieren por una serie de méritos después de haber sido juez y que ejercen en el seno de órganos colegiados. Por lo tanto, además de esa diferencia que existe con el hecho de que el juge des libertés et de la détention sea una figura inexistente en el sistema penal español, nos encontramos que, de entrada, juge y juez no son equivalentes absolutos.

Por todos los motivos que aquí se exponen, hemos optado por la técnica de traducción perifrástica, ya que es de la única forma, a nuestro parece, que el lector español que desconozca el sistema penal francés pueda comprenderlo de manera correcta.

\subsection{MAGISTRAT}

\section{Explicación:}

Según Cornu (1987), podemos definir magistrat de la siguiente manera:

Au sens strict, toute personne appartenant au *corps judiciaire et investie, à titre professionnel, du pouvoir de rendre la justice ( ${ }^{*}$ magistrat du siège) ou de le requérir au nom de l'État ('magistrat du parquet). Ne pas confondre avec *juge [...] (Cornu, 1987: 631).

Es decir, en Francia, el término magistrat hace referencia a cualquier persona que desempeña una autoridad pública. No obstante, dentro de un contexto procesal, podemos encontrar las siguientes denominaciones:

- Magistrat debout o du parquet: representa y defiende a la sociedad. Asimismo, dirige la acción de la policía judicial, entre otras. Es quien propone una pena al juez en nombre de la sociedad. Se encuentra bajo la dependencia del Garde des Sceaux (ministro de la justicia francés).

- Magistrat du siège: es independiente e inamovible. Soluciona los conflictos que se produce entre las personas (como, por ejemplo, conflictos de familia, vivienda, consumo, etc.). Igualmente, castiga a los responsables de los delitos y garantiza los intereses de las víctimas y la sociedad. Sus decisiones siempre van de acuerdo con la ley y es responsable del buen funcionamiento de las pruebas. 


\section{Contexto:}

Nicolas Sarkozy, antiguo presidente de Francia, fue detenido por, presuntamente, hacer una financiación ilegal, procedente de libia, de su campaña presidencial de 2007. Por este motivo, es el magistrat Tournaire quien está a cargo de la investigación de estas sospechas.

\section{Propuesta de traducción:}

Juez

Técnica de traducción empleada:

Equivalente funcional y equivalente contextual

\section{Comentarios:}

Antes de nada, consideramos oportunos hacer una diferencia entre juez y magistrado en español, pues sin su correcta comprensión, la traducción de magistrat puede verse distorsionada por errores de traducción, tales como el falso sentido. En España se dividen los órganos jurisdiccionales en unipersonales y colegiados. Hablamos de órganos unipersonales cuando solo hay una persona que juzga (en este caso, los órganos se denominan Juzgados) y, por el contrario, hablamos de órganos colegiados cuando el veredicto corre a cargo de un grupo de personas, un colegio. A esas personas juzgadoras se les denomina magistrado y los cargos donde cumplen su función los magistrados se denomina Tribunal. No obstante, el derecho francés hace una distinción en cuanto al rango al que pertenecen (independientemente de su composición). Así, podemos diferenciar entre cour y tribunal, siendo el primero aquel órgano de rango superior $y$, el segundo, el de rango inferior. Esto supone ya un anisomorfismo cultural, porque no vamos a traducir juge o magistrat en función de si los órganos son colegiados o unipersoneles. En este caso, hemos realizado una investigación acerca del magistrat del caso (Serge Tournaire). Trabaja en el Tribunal de grande instance, que es un órgano en el que solo una persona es la juzga, unipersonal, por lo que, para evitar confusiones (a pesar de la diferencia existente entre ambas realidades), optamos por traducir (en este contexto) magistrat por juez.

Asimismo, vemos que en francés hace mención también al magistrat débout; es decir, lo que sería nuestro fiscal (que también, en francés, se le denomina Parquet o Procureur). Con lo cual, en otros contextos, no podríamos ni traducirlo por juez ni por magistrado. Es por este motivo que el conocimiento de la realidad cultural, tanto en España 
como en Francia, resulta primordial. Debemos conocer de qué tipo de órganos se está hablando y a quién se hace referencia (la fase documental será crucial) y, al mismo tiempo, saber todo el contenido del ámbito en la lengua meta para poder darle la traducción apropiada. Vemos, una vez más, que no se da un equivalente total entre ambos idiomas y que la importancia de los conocimientos culturales de ambos países es clave en el proceso traslativo.

\subsection{PARQUET}

\section{Explicación:}

Según la página del gobierno francés Vie public el parquet hace referencia a la organización del tribunal de grande instance, del conjunto de los magistrats del ministerio (francés) público que se encargan de solicitar la aplicación de la ley y llevar a cabo un proceso penal en nombre de los intereses de la sociedad. Es importante no confundir este término (como se suele hacer a menudo) como sinónimo del Ministère public (Ministerio Fiscal), que se trata del órgano que presenta y sostiene una acusación pública en el proceso penal. El parquet sería, en este caso, la unidad administrativa a la que pertenecen las personas que representan el órgano mencionado anteriormente.

No obstante, en cada tribunal de grande instance, hacemos referencia al parquet cuando hablamos de la figura francesa del fiscal, que representa al ministerio. El parquet tiene la misión de velar por la aplicación de la ley en nombre del respeto de los intereses fundamentales de la sociedad.

\section{Contexto:}

Naomi Musenga es una joven que vivía en Estrasburgo y que llamó un día al Service d'aide médicale urgente ${ }^{3}$ (SAMU) porque se encontraba mal, con dolores muy intensos en el vientre y con fiebre. Las grabaciones de la llamada muestran cómo la operadora no la toma en cuenta e incluso hay momentos de burlas hacia la persona de Naomi. La operadora le dice que llame al médico y se despide. Es importante saber que Naomi tuvo el coraje de llamar al médico, pero que murió seis horas después de su llamada al SAMU por disfunción multiorgánica. Por todos estos motivos,

\footnotetext{
${ }^{3}$ El servicio de urgencias médicas francés.
} 
el Parquet de Estrasburgo ha decidido abrir una investigación de los hechos.

Propuesta de traducción:

Fiscalía

Técnica de traducción empleada:

Equivalente funcional y equivalente contextual

\section{Comentarios:}

En primer lugar, como traductores, debemos prestar mucha atención a la polisemia que presenta esta palabra. El contexto es quien nos va a indicar si debemos optar por una traducción u otra. En este caso, consideramos que se hace referencia a lo que en español denominamos Fiscalía, porque entendemos que en este contexto no se hace referencia al agente, sino a la unidad administrativa. Si hacemos, asimismo, hincapié en el sentido de este contexto, debemos saber que en Francia el Parquet depende directamente del Garde des Sceaux (expresión que se usa en Francia para nombrar a su Ministro de Justicia), mientras que en España los fiscales están bajo dependencia directa del Fiscal General del Estado. De entrada, he aquí el primer anisomorfismo cultural. Por ese motivo, no podemos afirmar que ambos términos (el francés y el español) sean equivalentes totales. Además, como hemos mencionado al principio de este subapartado de comentarios, la palabra Parquet posee una dualidad en cuanto a su terminología (a diferencia del español). De ahí que en la técnica de traducción expongamos que utilizamos también un equivalente contextual. Cuando se trata del funcionario adscrito a la fiscalía (en España, Fiscal), dijimos que también se denominaba Parquet. Ahora bien, según el nivel de los tribunales, podemos hablar de los siguientes conceptos:

- Procureur Général siempre y cuando se encuentre adscrito a las instancias jurisdiccionales superiores; es decir, Cour de Cassation, Cour d'Assises o Cour d'Appel).

- Procureur de la République siempre y cuando se encuentre adscrito al Tribunal Correctionnel.

Por lo tanto, podemos ver que, a pesar de la «similitud» de los conceptos en ambos idiomas, la realidad cultural que se esconden tras ellos es muy diferente. Si un francés que maneje el derecho lee Parquet 
como traducción de Fiscal o Fiscalía, no va tener presente la misma realidad que la del lector de la lengua original ( $y$ viceversa).

\subsection{TRIBUNAL CORRECTIONNEL}

\section{Explicación:}

Antes de explicar lo que es el Tribunal correctionnel en sí, consideramos oportuno conocer el significado de la palabra tribunal.

Según Cornu (1987), en su segunda acepción, tribunal sería lo siguiente:

La juridiction elle-même, que celle-ci soit composée de plusieurs magistrats (tribunal de grande instance) ou d'un seul (tribunal d'instance). Parfois cependant, tribunal, employé seul, désigne plutôt une juridiction collégiale qu'un juge unique (Cornu, 1987: 1042).

Es decir, se trata de una jurisdicción (ya sea compuesta por uno o más magistrats), aunque, cuando la palabra tribunal se emplea sola, puede designar más bien a una jurisdicción colegiada.

En lo concerniente a Tribunal correctionnel, la página web del Ministerio de Justifica de Francia nos explica que es aquel órgano que juzga casos como el robo o el abuso de confianza que cometen aquellas personas mayores de edad. Asimismo, juzga aquellos delitos leves que están ligados a un delito menos leve; por ejemplo, el hecho de herir a un peatón (esto sería un delito menos leve) por conducir con exceso de velocidad (esto será un delito leve).

En definitiva (y grosso modo), se trata de aquel órgano que es competente para el enjuiciamiento de los delitos leves.

\section{Contexto:}

En Rennes, el Tribunal correctionnel examina varios casos de delitos en la carretera. Este órgano juzga a algunas personas incluso por caso reincidente. Uno de los investigados confirma que un día estaba en un estado de embriaguez tal, que vomitaba mientras conducía y que lo veía todo negro.

Propuesta de traducción:

Tribunal correccional 


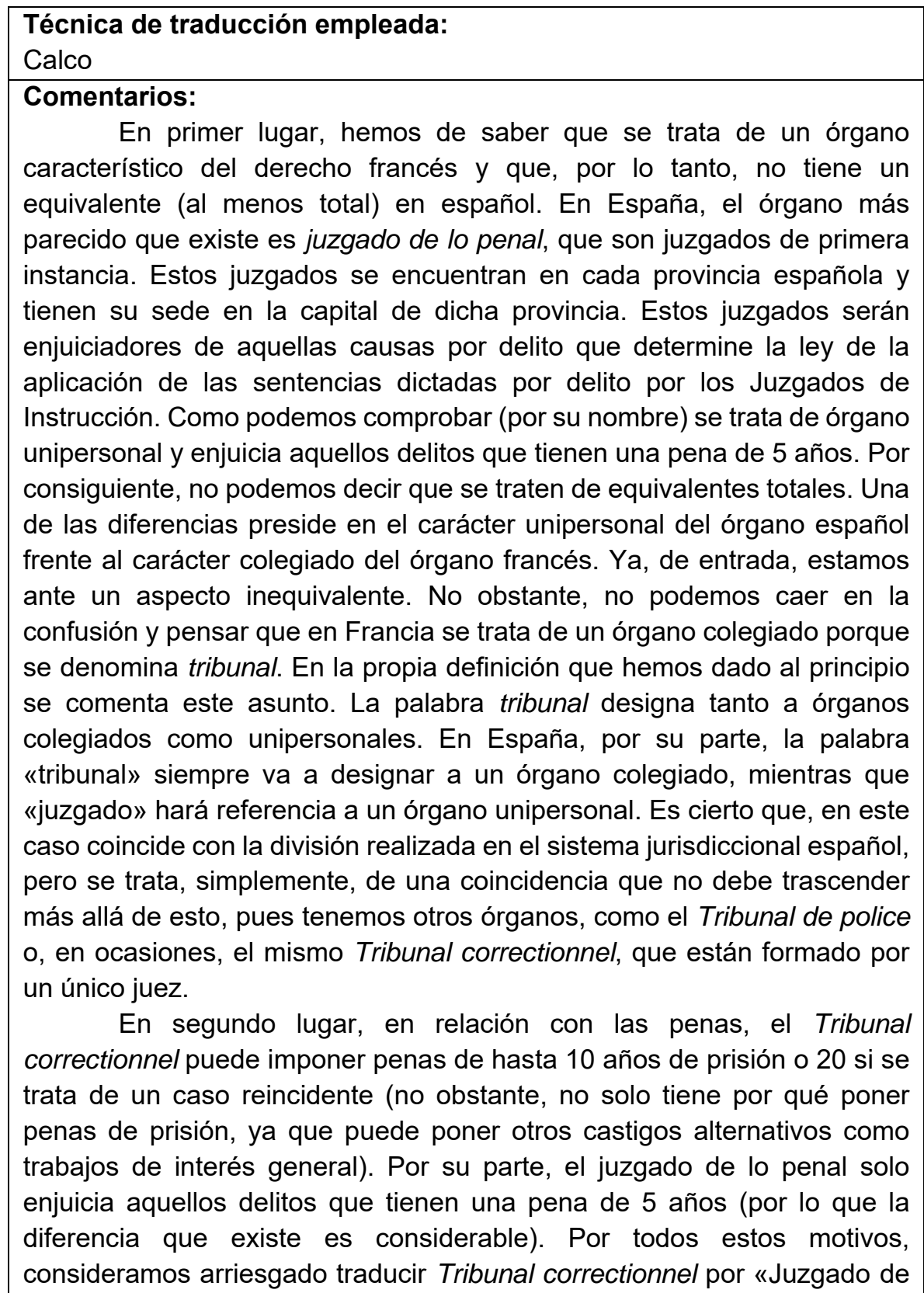

Hikma 18 (1) (2019), 231 - 260 
lo penal». Podríamos, en todo caso, traducirlo por «Juzgado de lo penal [francés], de forma que dejáremos claro que es un organismo distinto al español, pero, a nuestro entender, se trata de una propuesta ambigua para lector de la lengua, quien puede pensar que se trata de un órgano unipersonal.

\section{CONCLUSIONES}

En primer lugar, podemos afirmar que el conocimiento de las realidades culturales juega un papel fundamental en la figura del traductor $y$, en concreto, del traductor jurídico. Consideramos que debe ser un aspecto que se tiene que tratar con especial atención en los diferentes planes de estudios de Traducción e Interpretación. Este hecho es uno de los mayores problemas a los que se debe enfrentar el traductor jurídico (sobre todo, el estudiante de la asignatura de traducción jurídica). Normalmente, los alumnos de grado o máster se enfrentan a textos de una dificultad considerable sin conocer, de entrada, la realidad del país de la lengua materna (cuanto menos la realidad del país de la lengua extranjera). Podría ser algo bastante positivo que los alumnos, antes de iniciarse en la asignatura de traducción jurídica, tuviesen asignaturas de derecho comparado, lo cual evitaría muchos de los problemas que hay hoy en día.

En segundo lugar, a pesar de lo que acabamos de mencionar, debemos afirmar que es imposible que una persona con una formación en traducción (aunque tomara cursos de derecho comparado) sepa tanto como una persona con estudios de derecho. Por ese motivo, otros de los aspectos en los que se debería incidir es en la documentación. Se trata, a nuestro parecer, de uno de los procesos más fundamentales y en los que se debería dedicar un tiempo considerable antes de iniciarse en lo que es la trasposición de un idioma a otro. Si se hace una buena documentación, fase clave en el proceso traslativo, el resto de etapas serán mucho más llevaderas y con un menor grado de dificultad. Además, en el ámbito jurídico, la fase de documentación se vuelve un poco más complicada por varias razones:

- El lenguaje jurídico hace que la comprensión de lo que se quiere descubrir sea mucho más lenta que en cualquier otro ámbito

- La constante actualización de los sistemas penales, civiles, etc., hace que no todo lo que se lea por internet o por un manual sea correcto, pues ha podido sufrir variaciones de las que debemos ser conocedores a la hora de traducir. 
Por todo ello, creemos que para asimilar este anisomorfismo, también sería pertinente que el traductor estuviese en contacto con informaciones relativas al ámbito en cuestión (en lo concerniente a este trabajo sería el ámbito penal). Leer prensa sería una muy buena base, pero habría de complementarla con documentos oficiales. Tener curiosidad cultural es algo que, como en todo tipo de traducciones, debe estar presente en el traductor para poder evolucionar cada día más. Esto ya es algo que no depende tanto de las instituciones académicas, sino del propio estudiante en Traducción o el traductor. Se trata de un gran trabajo que hay que realizar día a día y poco a poco, pero que, sin duda, dará sus frutos.

$Y$, en último lugar, debemos confirmar que el anisomorfismo cultural en la terminología penal (francés-español) es una realidad. Esto hace que se dificulte la labor traslativa, pues la búsqueda de un equivalente no va a ser tan evidente como en otro tipo de ámbitos donde la diferencia de realidades es menor. No obstante, debemos, igualmente, afirmar que la traducción, desde nuestro punto de vista, es posible; es posible traducir realidades inequivalentes. Para ello, habrá que valerse de una serie de técnicas (por ejemplo, las expuestas en este trabajo). En función del grado anisomórfico, se tendrá que utilizar unas técnicas u otras.

En definitiva, y por las razones antes evocadas, la consideración del factor anisomórfico en traducción jurídica por parte del traductor es de vital importancia, pues, a pesar de la cercanía de ambos países, estamos frente a dos sistemas jurídicos que son totalmente diferentes. Además, es importante también incidir en la importancia que juega la documentación en traducción y los aspectos que se deben tener en cuenta a la hora de documentarse para traducir un texto de índole jurídica (por ejemplo, las constantes modificaciones que se suele hacer en las normas jurídicas). Y, finalmente, a pesar de estar frente a dos realidades totalmente distintas, podemos confirmar que, gracias al uso de las correctas técnicas de traducción, la traducción de dichas realidades anisomórficas sí es posible.

\section{REFERENCIAS BIBLIOGRÁFICAS}

Alcaraz Varó, E. y Hugues, B. (2001). El español jurídico. Barcelona, España: Ariel (Actualización a cargo de Adelina Gómez, 2009).

Arntz, R. y Picht, H. (1989). Introducción a la terminología. Madrid, España: Ediciones Pirámide.

Bocquet, C. (2008). La traduction juridique : Fondement et méthode. Bruselas, Bélgica: De Boeck. 
Boletín Oficial del Estado (BOE) (2018). Ley Orgánica 10/1995, de 23 de noviembre, del Código Penal. Recuperado de https://www.boe.es/buscar/act.php?id=BOE-A-1995-25444 [Fecha de consulta: 15-1-2018]

Boletín Oficial del Estado (BOE) (2018). Real decreto de 14 de septiembre de 1882 por el que se aprueba la Ley de Enjuiciamiento Criminal. Recuperado de https://www.boe.es/buscar/act.php?id=BOE-A-18826036 [Fecha de consulta: 15-1-2018]

Camille Jauffret-Spinosi, R. D. (1992). Les grands systèmes de droit contemporains. París, Francia: Dalloz.

Campos Plaza, N. (2010). Équivalents terminologiques des organes judiciaires et de l'ordre juridictionnel français et espagnol. Anales de Filología Francesa, 18, pp. 71-84.

Catford, John Cunnison (1965). A linguistic Theory of Translation: An Essay in Applied Linguistics. Londres, Reino Unido: Oxford University Press (Una teoría lingüística de la traducción: ensayo de lingüística aplicada. Caracas, Venezuela: Universdiad Central de Venezuela, 1970).

Centre National De Ressources Textuelles Et Lexicales (2018). Recuperado de http://www.cnrtl.fr/definition/. [Fecha de consulta: 1-3-2018].

Cornu, G. (1987). Vocabulaire juridique. París, Francia: Quadrige.

Dictionnaire Juridique (2018). Dictionnaire du droit privé. Recuperado de https://www.dictionnaire-juridique.com/. [Fecha de consulta: 02-052018].

Droit.FR (2018). Definitions juridiques. Recuperado de https://www.droit.fr/. [Fecha de consulta: 26-5-2018].

Enciclopedia Jurídica (2018). Diccionario de Derecho. Recuperado de http://www.enciclopedia-juridica.biz14.com/inicio-enciclopediadiccionario-juridico.html. [Fecha de consulta: 16-5-2018].

Gutiérrez Arcones, D. (2015): Estudio sobre el texto jurídico y su traducción: características de la traducción jurídica, jurada y judicial. Miscelánea Comillas, 142, pp. 141-175.

Holl, I. (2012). Técnicas para la traducción jurídica: revisión de diferentes propuestas, últimas tendencias. Hermeneus, 14, pp. 191-216.

Hurtado Albir, A. (2011). Traducción y Traductología: Introducción a la Traductología. Madrid, España: Cátedra. 
Junta de Andalucía (2018). Portal Adriano. Consejería de Justicia e Interior. Recuperado de http://www.juntadeandalucia.es/justicia/portal/adriano/ admonjusticia/justiciaenandalucia/juzgados/. [Fecha de consulta: 20-52018].

Justicia en Navarra (2018). La administración de la justicia. Recuperado de http://www.navarra.es/NR/rdonlyres/E0F960C2-C5B8-45EC9ED529A3BD05DD81/121526/Justiciaad. [Fecha de consulta: 2-42018].

Karim, H. (2011). El concepto de anisomorfismo cultural en el ámbito de jurídico: concepto y aplicaciones traductológicas sobre la legislación española en comparación con la marroquí. EntreCulturas, 2, pp. 99105.

Köller, W. (1995). The Concept of Equivalence and the Object of Translation Studies. Target, 7/2, pp. 191-222.

Larousse (2018). Dictionnaire de français. Recuperado de https://www.larousse.fr/dictionnaires/francais. [Fecha de consulta: 265-2018].

Legifrance (2018). Code de procédure pénal. Recuperado de https://www.legifrance.gouv.fr/affichCode.do?cidTexte=LEGITEXT000 006071154\&dateTexte=29990101 [Fecha de consulta: 15-1-2018].

Legifrance (2018). Code pénal. Recuperado de https://www.legifrance.gouv.fr/affichCode.do?cidTexte=LEGITEXT000 006070719\&dateTexte=20180528 [Fecha de consulta: 15-1-2018].

Lobato Patricio, J., (2009). La traducción jurídica, judicial y jurada: vías de comunicación con las administraciones. EntreCulturas, 1, pp. 191-206.

Ministère de la justice (2018). Juge des libertés et de la détention. Recuperado de http://www.justice.gouv.fr/organisation-de-la-justice10031/lordre-judiciaire-10033/juge-des-libertes-et-de-la-detention25302.html. [Fecha de consulta: 22-5-2018].

Ministère de la justice (2018). Métiers. Recuperado de http://www.metiers.justice.gouv.fr/magistrat-12581/le-metier-12582/. [Fecha de consulta: 18-5-2018].

Ministere de la justice (2018). Présentation de l'ordre judiciaire. Recuperado en http://www.justice.gouv.fr/organisation-de-la-justice-10031/lordrejudiciaire-10033/. [Fecha de consulta: 2-3-2018]. 
Nida, E. A. (1975). Language Structure and Translation. Stanford, Estados Unidos: Stanford University Press.

Orozco Jutorán, M. (2014). «Propuesta de un catálogo de técnicas de traducción: la toma de decisiones informada ante la elección de equivalentes». Hermeneus, 16, pp. 233-264.

Peñaranda López, A. (2015). Proceso Penal Comparado (España, Inglaterra, Estados Unidos, Rusia). Descripción y terminología. Albolote (Granada), España: Comares.

Pérez Guarnieri, V. F. (2017). La práctica de la traducción jurídica: problemas y dificultades en la traducción de sentencias (inglés-español) en dos casos de derecho penal y civil. (Tesis doctoral). Universidad de Córdoba, Córdoba: UCOPress.

Poder Judicial (2018). Poder Judicial España. Recuperado de http://www.poderjudicial.es/cgpj/es/Poder_Judicial. [Fecha de consulta: 2-3-2018].

Real Academia de la Lengua Española (2018). Diccionario de la Lengua Española. Recuperado de http://dle.rae.es/?w=diccionario. [Fecha de consulta: 2-10-2018].

Šarčević, S. (1997). New Approach to Legal Translation. La Haya, Londres, Boston: Kluwer Law International.

Service Public (2018). Le site officiel de l'administration française. Recuperado en https://www.service-public.fr/. [Fecha de consulta: 155-2018].

Tricás Preckler, M. (2003). Manual de traducción. Barcelona, España: Gedisa Editorial.

Vie Public (2018). Vie public au coeur du débat public. Recuperado de http://www.vie-publique.fr/. [Fecha de consulta: 15-5-2018]. 